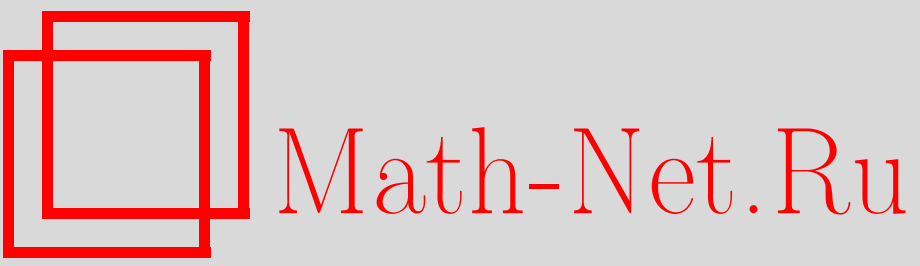

А. Л. Скубачевский, О некоторых свойствах эллиптических и параболических функционально-дифференциальных уравнений, УМН, 1996, том 51, выпуск 1, 169-170

DOI: https://doi.org/10.4213/rm937

Использование Общероссийского математического портала Math-Net.Ru подразумевает, что вы прочитали и согласны с пользовательским соглашением

http://www . mathnet.ru/rus/agreement

Параметры загрузки:

IP : 3.82 .47 .9

26 апреля 2023 г., 13:32:41 


\section{О НЕКОТОРЫХ СВОЙСТВАХ ЭЛЛИПТИЧЕСКИХ И ПАРАБОЛИЧЕСКИХ ФУНКЦИОНАЛЬНО-ДИФФЕРЕНЦИАЛЬНЫХ УРАВНЕНИЙ}

\section{А. Л. СКУБАЧЕВСКИЙ}

В заметке рассматривается квазилинейное параболическое функционально-дифференциальное уравнение, возникающее в нелинейной оптике [1]. Результаты данной работы о стационарных решениях этого уравнения дают необходимые условия существования бифуркаций. Один из подходов к исследованию бифуркаций основан на спектральных свойствах линеаризованного эллиптического функционально-дифференциального оператора. Получены необходимые и достаточные условия существования ортонормированного базиса, состоящего из собственных функций этого оператора.

Рассмотрим квазилинейное параболическое уравнение

$$
u_{t}=D \Delta u-u+K\left(1+\gamma \cos u_{g}\right) \quad(x \in Q, t>0) .
$$

Здесь $Q \subset \mathbb{R}^{2}$ - ограниченная область с границей $\partial Q \in C^{\infty} ; D, \gamma>0, K \neq 0$ - вещественные числа; $D$ - коэффициент диффузии, $\gamma$ - видность интерференционной картины, $K$ - коэффициент, пропорциональный интенсивности входного поля; $u=u(x, t)$ - фаза световой волны, $u_{g}=u(g(x), t) ; g$ - невырожденное преобразование класса $C^{3}$, отображающее ограниченную область $\Omega \subset \mathbb{R}^{2}(\bar{Q} \subset \Omega)$ на $g(\Omega)$ так, что $g(Q) \cap Q \neq \varnothing, g(x) \not \equiv x$.

Краевое условие имеет вид

$$
\left.\frac{\partial u}{\partial \widetilde{\nu}}\right|_{\partial Q \times(0, \infty)}=0
$$

где $\nu$ - единичный вектор внешней нормали к $\partial Q$ в точке $x, \widetilde{\nu}=(\nu, 0)$.

Поскольку в функционально-дифференциальном уравнении (1), вообще говоря, $g(Q) \backslash Q \neq \varnothing$, задается дополнительно следующее условие:

$$
u(g(x), t)=0 \quad(x \in Q: g(x) \notin Q ; t>0) .
$$

Очевидно, если $g(Q) \subset Q$, условие (3) не задается.

Стационарным решением задачи (1)-(3) мы назовем решение, не зависяшее от $x$ и $t$ в цилиндре $Q \times(0, \infty)$. Очевидно, такие решения должны удовлетворять следующим условиям:

$$
\begin{array}{ll}
w(x)=K[1+\gamma \cos w(g(x))] & (x \in Q), \\
w(g(x))=0 & (x \in Q: g(x) \notin Q), \\
w(x)=\mathrm{const} & (x \in Q) .
\end{array}
$$

Из определения стационарного решения легко получить следующее утверждение.

TeOPEMA 1. Пусть $g(Q) \backslash Q \neq \varnothing$.

Тогда условие $K(1+\gamma)=2 \pi n \quad(n= \pm 1, \pm 2, \ldots)$ является необходимьм и достаточным для разрешимости задачи (4)-(6). Если $K(1+\gamma)=2 \pi n \quad(n= \pm 1, \pm 2, \ldots)$, задача (4)-(6) имеет единственное решение $w(x)=K(1+\gamma)$.

Обозначим через $\widehat{w}$ некоторое решение задачи (4)-(6) при $K=\widehat{K}$. Положим $u(x, t)=$ $\widehat{w}+v(x, t)$. Тогда

$$
v_{t}=L v+N(v)
$$

Работа выполнена при финансовой поддержке Российского фонда фундаментальных исследований (грант № 95-01-00247). 
где $L v=D \Delta v-v-\widehat{K} \gamma \sin \widehat{w} v_{g}, N(v)=\widehat{K} \gamma\left\{\cos \left(\widehat{w}+v_{g}\right)-\cos \widehat{w}+v_{g} \sin \widehat{w}\right\}$.

Рассмотрим линеаризованный оператор $L: L_{2}(Q) \rightarrow L_{2}(Q)$ с областью определения $D(L)=$ $\left\{v \in W_{2}^{2}(Q):\left.\frac{\partial v}{\partial \nu}\right|_{\partial Q}=0\right\}$, действующий по формуле $L v=D \Delta v-v-\widehat{K} \gamma \sin \widehat{w} v_{g}$. Здесь $W_{2}^{k}(Q)$ - пространство Соболева функций из $L_{2}(Q)$, имеющих все обобщенные производные до поря дка $k$ включительно из $L_{2}(Q)$.

Как известно, существование бифуркаций связано с наличием пары простых комплексно сопряженных чисто мнимых собственных значений оператора $L$ и непрерывным изменением стационарного решения при непрерывном изменении параметра $K$. В случае $g(Q) \backslash Q \neq \varnothing$ стационарные решения сушествуют лишь при изолированных значениях коэффициента $\widehat{K}=2 \pi n(1+\gamma)^{-1}$ $(n= \pm 1, \pm 2, \ldots)$, а линеаризованный оператор $L=D \Delta v-v$ является самосопряженным. Следовательн, при $g(Q) \backslash Q \neq \varnothing$ задача (1)-(3) не имеет бифуркационного семейства периодических решений. Поэтому в дальнейшем будем предполагать, что $g(Q) \subset Q$. Обозначим $G=\left\{x \in Q: g^{2}(x) \neq x\right\}$.

Teоpema 2. Пусть $g(Q) \subset Q, \widehat{w} \neq \pi n \quad(n= \pm 1, \pm 2, \ldots)$ u nусть $G \neq \varnothing$.

При этих предположениях собственные функции оператора L составляют ортонормированный базис в $L_{2}(Q)$ тогда и только тогда, когда $g(Q)=Q$ и $g(x)=A x+b$ $(x \in Q)$, где $A=\left(\begin{array}{cc}\cos \theta & -\sin \theta \\ \sin \theta & \cos \theta\end{array}\right), 0<\theta<2 \pi, \theta \neq \pi, b \in \mathbb{R}^{2}$.

Teорема 3. Пусть $g(Q) \subset Q, \widehat{w} \neq \pi n \quad(n= \pm 1, \pm 2, \ldots)$ u nусть $G=\varnothing$.

При этих предположениях собственные функции оператора L составляют ортонормированный базис в $L_{2}(Q)$ тогда и только тогда, когда $g(Q)=Q$ u $\left|J_{g}(x)\right| \equiv 1$ $(x \in Q)$, әде $J_{g}(x)=\left|\begin{array}{ll}g_{1 x_{1}}(x) & g_{1 x_{2}}(x) \\ g_{2 x_{1}}(x) & g_{2 x_{2}}(x)\end{array}\right|, g(x)=\left(g_{1}(x), g_{2}(x)\right)$.

В заключение отметим, что существование бифуркационного семейства решений задачи (1), (2) спектральные свойства оператора $L$ рассматривались в работе [2] в случае, когда $Q=\left\{x \in \mathbb{R}^{2}:|x|<1\right\}$, а для $g(x)$ - преобразование поворота на угол $0<\theta<2 \pi$. Некоторые вопросы спектральной теории для эллиптических функционально-дифференциальных операторов изучались в [3], [4].

\section{СПИСОК ЛИТЕРАТУРЫ}

[1] Воронцов М. А., Думаревский Ю. Д., Пруидзе Д. В., Шмальгаузен В. И. // Изв. АН СССР. Физика. 1988. Т. 52 № 2. С. 374-376. [2] Razgulin A. V. // Chaos in Optics / ed. Rajarshi Roy. Proceedings SPIE 2039, 1993. P. 342-352. [3] Skubachevskii A. L. // J. Differential Equations. 1986. V. 63. № 3. Р. 332-361. [4] Скубачевский А. Л., Цветков Е. Л. // Дифференц. уравнения. 1989. Т. 25. № 9. С. 1766-1776. 